\title{
Investigating bacterial population structure and dynamics in traditional koumiss from Inner Mongolia using single molecule real-time sequencing
}

\author{
Qimu Gesudu, ${ }^{*}{ }^{1}$ Yi Zheng, ${ }^{*}{ }^{1}$ Xiaoxia $X i,{ }^{*} \dagger$ Qiang Chuan Hou, ${ }^{*} \dagger$ Haiyan $\mathrm{Xu},{ }^{*} \dagger$ Weiqiang Huang, ${ }^{*} \dagger$ \\ Heping Zhang, ${ }^{*} †$ Bilige Menghe, ${ }^{*} \dagger$ and Wenjun Liu* ${ }^{2}$ \\ *Key Laboratory of Dairy Biotechnology and Engineering, Ministry of Education, Inner Mongolia Agricultural University, Huhhot, China 010018 \\ †Dairy Processing Laboratory of National Dairy Production Technology and Research Center, Huhhot, Inner Mongolia, China, 010018
}

\begin{abstract}
Koumiss is considered as a complete dairy product high in nutrients and with medicinal properties. The bacterial communities involved in production of koumiss play a crucial role in the fermentation cycle. To reveal bacterial biodiversity in koumiss and the dynamics of succession in bacterial populations during fermentation, 22 samples were collected from 5 sampling sites and the full length of the $16 \mathrm{~S}$ ribosomal RNA genes sequenced using single molecule real-time sequencing technology. One hundred forty-eight species were identified from 82 bacterial genera and 8 phyla. These results suggested that the structural difference in the bacterial community could be attributed to geographical location. The most significant difference in bacterial composition occurred in samples from group D compared with other groups. The sampling location of group D was distant from the city and maintained the primitive local nomadic life. The dynamics of succession in bacterial communities showed that Lactobacillus helveticus increased in abundance from 0 to $9 \mathrm{~h}$ and reached its peak at $9 \mathrm{~h}$ and then decreased. In contrast, Enterococcus faecalis, Enterococcus durans, and Enterococcus casseliflavus increased gradually throughout the fermentation process, and reached a maximum after $24 \mathrm{~h}$.
\end{abstract}

Key words: koumiss, biodiversity, predominant species, SMRT sequencing, fermentation succession

\section{INTRODUCTION}

Koumiss (kumis), also called airag, arrag, and chige in the Mongolian language, is a fermented mare milk beverage that is popular in Central Asia and Eastern Europe. The production of the ancient beverage koumiss can be traced to the ancient Greeks and Romans in Europe (Wszolek et al., 2006). In China, koumiss

Received March 14, 2016.

Accepted July 3, 2016.

${ }^{1}$ These two authors contributed equally to the study.

${ }^{2}$ Corresponding author: wjliu168@163.com also has a long history as a popular drink among traditional nomadic people. The earliest record of koumiss appeared during the Han dynasty (202 BC-202 $\mathrm{AD})$ and its popularity became widespread during the Yuan dynasty (1271 AD-1368 AD; Zhang and Zhang, 2012). Koumiss has long been considered as a complete food, rich in nutrients with medicinal properties. In the 18th century, koumiss was thought to have therapeutic value in the treatments of wasting diseases caused by phthisis, abdominal tumors, and dyspepsia (Thompson and Leamnington, 1879). In recent years the Cossacks have introduced koumiss into military rations to prevent tuberculosis (Ishii and Samejima, 2001). Chen et al. (2010) found that koumiss is rich in angiotensin I-converting enzyme inhibitory peptides, which have antihypertensive properties. Chaves-López et al. (2012) also reported angiotensin I-converting enzyme inhibitory activity in yeast strains isolated from Colombian koumiss.

Traditionally, fermentation of koumiss was achieved in wooden casks or in bags made of animal skin (Zhang and Zhang, 2012). At present, porcelain urns may also be used. In general, koumiss is produced by fermentation of natural microflora, including lactic acid bacteria (LAB) and yeasts, at ambient temperatures (approximately $20^{\circ} \mathrm{C}$ ) for 1 to $3 \mathrm{~d}$ (Park et al., 2006; Sun et al., 2010). During koumiss manufacture, filtered fresh mare milk is poured into urns that are placed outside the Mongolian yurt, and beaten or stirred with a wooden stick to ensure mixing evenly and fast fermentation. Every day, after removing what is required for consumption, a small aliquot of koumiss is retained for use as a starter culture for the next day, and more milk is added to ensure ongoing fermentation.

The microorganisms responsible for fermentation of koumiss mainly consist of LAB and yeast. Due to the therapeutic effects of koumiss and the fact that it is regarded as a valuable natural source of LAB, interest has been growing in identifying the microflora in koumiss by culture-dependent and culture-independent methods. Much research has been done to identify the LAB community using pure culture methods and has 
Table 1. Samples of koumiss that had been fermented for different lengths of time and collected from different sites in Xinlingol

\begin{tabular}{llllll}
\hline Sample & Site 1 & Site 2 & Site 3 & Sites 4 & Site 5 \\
\hline Fresh mare milk & A1 ${ }^{1}$ & B1 & C1 ${ }^{1}$ & D1 & E1 ${ }^{1}$ \\
$0 \mathrm{~h}$ & A2 & B2 & C2 & D2 ${ }^{1}$ & E2 \\
$3 \mathrm{~h}$ & A3 & B3 & C3 & D3 & E3 \\
$6 \mathrm{~h}$ & A4 & B4 & C4 & D4 & E4 \\
$9 \mathrm{~h}$ & A5 & B5 & C5 & D5 & E5 \\
$12 \mathrm{~h}$ & A6 & B6 $6^{1}$ & C6 & D6 ${ }^{1}$ & E6 ${ }^{1}$ \\
$>24 \mathrm{~h}$ & - & B7 & - & - & - \\
\hline
\end{tabular}

${ }^{1}$ DNA was not successfully extracted, so sequencing was not possible for these samples.

suggested that the predominant LAB in koumiss is Lactobacillus and Lactococcus species (Oberman and Libudzisz, 1985). An et al. (2004) also found that the isolates in koumiss from Inner Mongolia were mainly Lactobacillus species. Sun et al. (2010) systematically investigated the Lactobacillus species in homemade koumiss in Xinjiang, Inner Mongolia, and Qinghai by the combined use of conventional and molecular methods. The overall distribution pattern demonstrated that the Lactobacillus groups from the 3 geographically distant regions varied significantly. Hao et al. (2010) studied bacterial biodiversity in traditional koumiss by denaturing gradient gel electrophoresis with similar results. However, the ratios of the most frequently isolated species varied between sampling sites. Culture-dependent methods are commonly used, but it is well recognized that these methods often fail to characterize (minor) populations of microorganisms that require selective enrichment. Furthermore, conventional methods are not able to detect noncultural species, which represents a serious limitation for identification of all species present in any given sample.

Pyrosequencing-based 16S ribosomal RNA (rRNA) profiling has emerged as a powerful technique to characterize the structure of microbial communities in ecological samples (Justé et al., 2008; Humblot and Guyot, 2009; Desai et al., 2010; Faveri et al., 2015). The single molecule real-time sequencing technology (SMRT) of Pacific Biosciences (Menlo Park, CA) is one of several next-generation sequencing technologies that are also currently in use. This new technology not only produces considerably longer and more accurate DNA sequences from individual unamplified molecules, but it is also as reliable and accurate as more traditional approaches (Bashir et al., 2012; Roberts et al., 2013). It can quickly and accurately identify bacteria to the species level. To understand the structure and population dynamics of bacterial communities in koumiss we used SMRT sequencing to characterize the species present in 22 samples of traditional koumiss collected at different times during the fermentation process, from 5 sampling sites in Xinlingol, Inner Mongolia.

\section{MATERIALS AND METHODS}

\section{Sample Collection}

A total of 31 traditional fermented koumiss samples were collected from 5 different sites in Xilingol, Inner Mongolia. The 5 sites involved were near the city (site 2) and far away from the city (site 4), as well as the family maintaining the original koumiss-making process (sites 1 and 5) and the small workshop-style production koumiss family (site 3 ). From the site where the family maintains the traditional making process, samples at different stages of fermentation were taken: fresh milk and $0,3,6,9,12$, and $>24 \mathrm{~h}$ of fermentation (Table 1). Samples were collected aseptically and were transported in a tank of liquid nitrogen. Among these, 9 samples were not evaluated further because we were unable to extract the DNA successfully for subsequent sequencing.

\section{DNA Extraction and PCR Amplification}

Genomic DNA was extracted from each sample using Qiagen DNA Stool Mini Kit (Qiagen, Hilden, Germany) following the manufacturer's instructions. The quality of extracted DNA was checked by $0.8 \%$ agarose gel electrophoresis and spectrophotometry (optical density at $260 \mathrm{~nm} / 280 \mathrm{~nm}$ ratio). All extracted DNA samples were stored at $-20^{\circ} \mathrm{C}$ before subsequent analysis.

Bacterial 16S rRNA gene was amplified by PCR for bar-coded pyrosequencing. The full-length sequences of the 16S rRNA gene of all bacteria were amplified using the universal forward 27F (5'-AGAGTTTGATCCTGGCTCAG- $3^{\prime}$ ) and the reverse 1541R (5'-AAGGAGGTGATCCAGCCGCA-3') primers (Devereux and Wilkinson, 2004). The PCR program was as follows: $95^{\circ} \mathrm{C}$ for $2 \mathrm{~min} ; 30$ cycles at $95^{\circ} \mathrm{C}$ for $30 \mathrm{~s}, 55^{\circ} \mathrm{C}$ for $30 \mathrm{~s}$, and $72^{\circ} \mathrm{C}$ for $30 \mathrm{~s}$, with a final extension of $72^{\circ} \mathrm{C}$ for $5 \mathrm{~min}$. The quality control for PCR amplification, sequence preprocessing, and raw data processing were performed as described previously (Quigley et al., 2012). 


\section{SMRT Pyrosequencing}

The PCR products from the amplified 16S rRNA genes were quantified using an Agilent DNA 1000 Kit and an Agilent 2100 Bioanalyser (Agilent Technologies) according to the manufacturer's instructions. The products of 50 amplicons were pooled together in equimolar ratios at a final concentration of $100 \mathrm{nmol} / \mathrm{L}$. The amplicons were sequenced using P6-C4 chemistry on a PacBio RS II instrument (Pacific Biosciences). The quality control for PCR amplifications and sequence preprocessing was performed following the methods of Mosher et al. (2013).

The protocol RS_ReadsOfinsert.1, which is available in the SMRT Portal version 2.7, was applied to process raw data. The restrictive filtering parameters were based on the following criteria: (1) minimum full passes was up to 5, (2) minimum predicted accuracy was 90, (3) 1,400 was the minimum read length of inserts, and (4) 1,800 was the maximum read length.

\section{Bioinformatics Processing}

The extraction of high-quality sequences was performed using the Quantitative Insights Into Microbial Ecology (QIIME[v1.7]) program and the UCLUST algorithm (Wareth et al., 2014) to align extracted high-quality sequences with under $100 \%$ clustering of sequence identity, and obtain representative sequences. The unique sequence set was classified into operational taxonomic units (OTU) under the threshold of $97 \%$ identity using UCLUST after selection of representative sequences. Chimera Slayer was used to remove potential chimeric sequences in the representative set of OTU (Amir et al., 2013). The taxonomy of each OTU representative sequence was performed using the Ribosomal Database Project II database and GREENGENS (Zhang et al., 2015) that classify groups at a minimum bootstrap threshold of $80 \%$. Operational taxonomic units that occurred only once or twice were discarded. A de novo taxonomic tree was constructed employing a representative chimera-checked OTU set in FastTree for downstream analysis (ADMI, 1971), including the $\beta$ diversity calculation. The Shannon-Wiener, Simpson's diversity, Chao1, and rarefaction estimators were calculated to evaluate the $\alpha$ diversity. UniFrac distance (Bounaadja et al., 2009) was based on the phylogenetic tree. Both weighted and unweighted calculations were performed for principal coordinate analysis.

\section{Statistical Analysis}

Statistical analyses were performed in the Matlab environment (The MathWorks Inc., Natick, MA) and using the software package Canoco for Windows 4.5 (Microcomputer Power, Ithaca, NY). Rarefaction analysis, Shannon diversity index, and Simpson's diversity index were used to estimate the richness and diversity of OTU. Principal coordinate analysis was used to clarify the structure of microbial communities in different samples. Differences in the relative abundances of taxonomic groups at phylum and genus levels between samples were evaluated using the Mann-Whitney test. $P$-values of less than 0.05 were considered significantly different between sample pairs. The graph presentations were generated by the $\mathrm{R}$ package version 3.1.2 and the Origin software package version 8.5.

\section{RESULTS AND DISCUSSION}

\section{Bacterial Sequence Abundance and Diversity}

A total of 109,227 full 16S rRNA gene sequences were generated from the 22 koumiss samples, with an average of 4,964 $(2,647-8,291)$ sequence reads for each sample. The sequences were submitted to the MG-RAST database in GenBank (reference numbers 4678699-4678720). Based on homologous sequence alignment methods and clustering with information extracted from the Ribosomal Database Project and Basic Local Alignment Search Tool databases, the lowest level of taxonomy of the identified OTU was determined (Table 2). Of the bacterial sequences, $0.4 \%$ could not be assigned to the genus level. For all samples, the Shannon diversity curves, but not the rarefaction curves, reached the saturation phase (Figure 1). This suggests that, although additional new phylotypes could possibly be identified by increasing the sequencing depth, the majority of bacterial phylotypes for the samples had already been captured in the analysis. The Shannon index, Simpson diversity index, Chao1, and observed species in each sample were used to evaluate species richness and diversity (Table 2). Because significant differences ( $P$ $<0.005)$ were observed for all indicators of bacterial community in the samples from all 5 sites, it can be concluded that a large difference was present in species richness and diversity of the bacterial community found at the different fermentation times.

\section{Analysis of the Bacterial Community in Koumiss}

Eight bacterial phyla were identified in the samples of koumiss in this study (Figure 1A), namely Firmicutes, Proteobacteria, Actinobacteria, Fusobacteria, Deinococcus-Thermus, TM6, Bacteroidetes, and Spirochaetes. The predominant phylum was Firmicutes (93.0\%), and the major bacterial genera (relative abundance of $>1 \%$ ) were Proteobacteria (4.6\%) and Acetinobacteria 

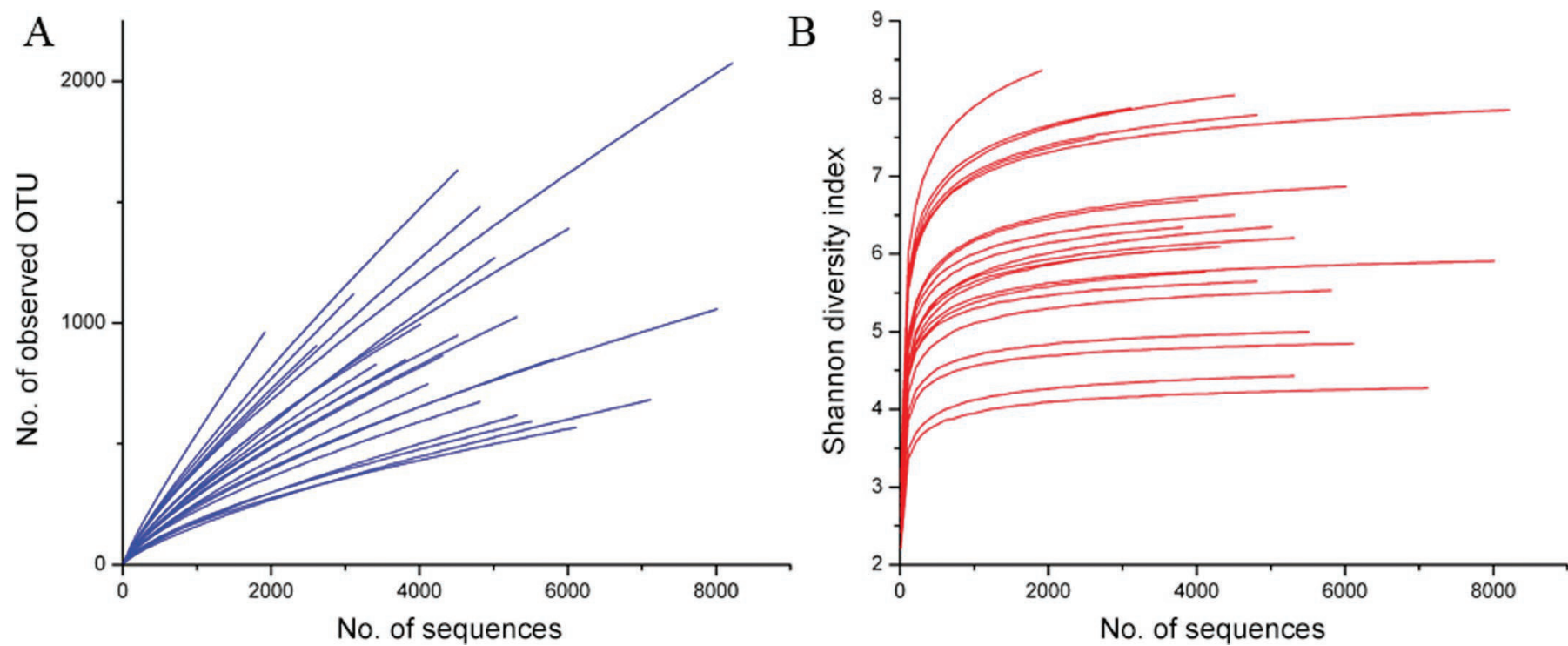

Figure 1. Shannon diversity curves and the rarefaction curves for the sample sequencing. OTU = operational taxonomic units. Color version available online.

(2.6\%). Eighty-two bacterial genera were identified The predominant (>10\%) genera were Lactobacillus, (Figure 1), including Lactobacillus (53\%), Lactococcus Lactococcus, and Streptococcus. The dominance of (21\%), Streptococcus (11\%), and Acetobacter (8.3\%). LAB, and particularly Lactobacillus species from the

Table 2. Sample information, sequence abundance, and microbial diversity of koumiss samples (mean $\pm \mathrm{SD}$ )

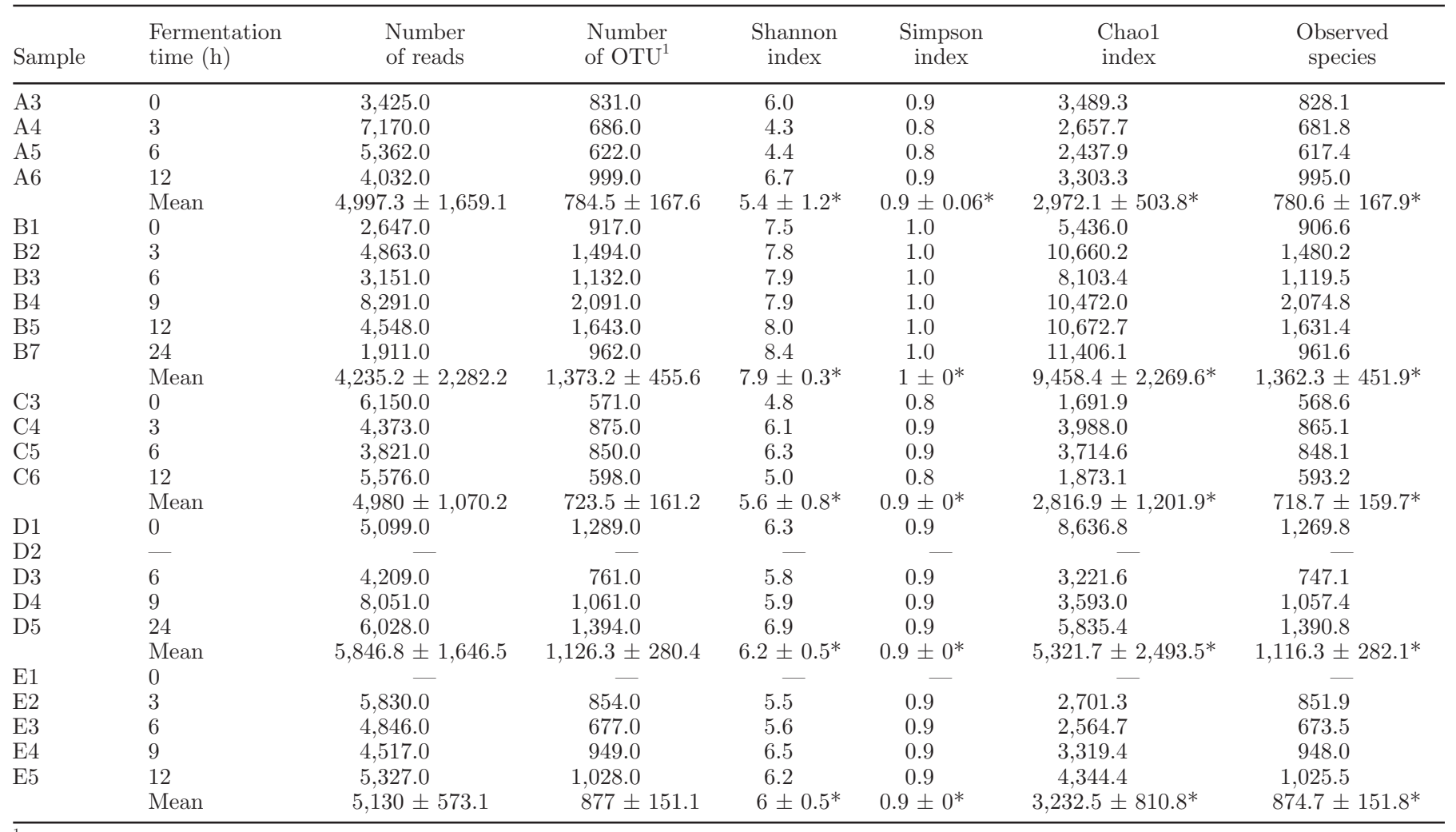

${ }^{1} \mathrm{OTU}=$ operational taxonomic units.

${ }^{*} P<0.05$ (by Mann-Whitney test) indicates significant differences between sample groups. 
phylum Firmicutes, is what would be expected from a traditionally produced fermented milk product (Hao et al., 2010; Schornsteiner et al., 2014; Zuo et al., 2014).

At the species level, 148 bacterial species were obtained. This represents considerably greater biodiversity compared with previous studies of koumiss that were based on cultivation or low-resolution molecular fingerprinting methods (Watanabe et al., 2008; Hao et al., 2010; Sun et al., 2010). The predominant species include Lactobacillus helveticus (56\%), Lactococcus lactis (21\%), Streptococcus parauberis (13.1\%), and Rothia nasimurium (8.4\%) showed in Figure 2. Lactobacillus helveticus, Lc. lactis, and S. parauberis were the predominant species in this study, which supported previous studies, although they only identified $L b$. helveticus as the predominant species (An et al., 2004; Uchida et al., 2007; Sun et al., 2010). Strains of Lactococcus, Streptococcus, and Enterococcus have also been isolated from koumiss in a few previous studies (An et al., 2004; Watanabe et al., 2008). However, the pyrosequencing method was generally faster, more specific, more sensitive, and more accurate, confirming that it is a more precise method for studying microbial diversity than earlier methods (Justé et al., 2008).

Variation in microbial diversity in different dairy products has been associated previously with geographic location and other environmental factors (Fuka et al., 2013; Sun et al., 2014). In the present study, significant differences were observed in bacterial species composition among the different groups of samples collected at each of the 5 sites (Figure 3). In the samples from site 1 (group A), Lc. lactis and Lb. helveticus were detected. Lactococcus lactis was regarded as the predominant species and was present throughout the entire fermentation process, whereas $L b$. helveticus was only detected in the late fermentation stage (A5, A6). Strains of $L b$. helveticus, S. parauberis, R. nasimurium, Lc. lactis, and unclassified strains were detected in samples from site 2 (group B). The most detected species were $\mathrm{Lb}$. helveticus and S. parauberis. Lactobacillus helveticus was the predominant species in samples from all the other sites (groups C, D, and E). Lactococcus lactis was most frequently detected in samples from site 4 (group D).

This was supported by Kruskal-Wallis analysis of the heat map which also showed differences in bacterial composition among the 5 sampling sites (Figure 4). Specifically, the relative abundance of Acetobacter pasteurianus, Lactobacillus hamsteri, Gluconacetobacter rhaeticus, and Enterococcus asini in samples from site 2 (group B) was significantly higher than that from the other sites.

The structure of the bacterial communities in the samples from the 5 different places was also compared using weighted and unweighted UniFrac principal co- ordinate analysis. Although some overlap was present in the score plots among the samples from the 5 sites, data points were largely separated in both weighted (accounting for 74.5 and $14.27 \%$ of the total variance by the first 2 principal components (PC), respectively; Figure 5A) and unweighted (accounting for 12.36 and $9.64 \%$ of the total variance by the first $2 \mathrm{PC}$, respectively; Figure 5B) analyses. The samples from sites 1 and 2 were significantly clustered separately and separated from all the other sites. These results suggest that differences in the bacterial communities could be associated with site location. Samples from site 2 had a certain tendency to cluster together in the lower right and upper left portion in Figure 5A and Figure 5B, respectively, which suggests that koumiss from these sampling sites have their own unique OTU at different fermentation times.

A further multivariate ANOVA test showed significant differences in the bacterial communities from the 5 different sites based on the weighted $(P<0.05)$ UniFrac analysis (Figure 6A). The unweighted analysis showed that the bacterial community structure was not significantly different among other groups except for site $2(P$ $>0.05$ ), and site 2 was significantly different from each other $(P<0.05)$ (Figure 6B). The most significant difference in bacterial composition was found in samples in group B compared with other groups (Figure 6A).

Overall the bacterial community structure varied between the 5 different sites. In particular, the bacterial structure at site 2 was significantly different from all the other sites. This site was distant from the city and maintained the primitive local nomadic life. The koumiss production process at this site was primitive and changed little over time, which could account for its unique and diverse bacterial community.

\section{Variation in Bacterial Communities During Fermentation}

Over the fermentation period (0-24 h), bacterial abundance increased as the fermentation time increased and the $\mathrm{pH}$ decreased. In particular, the relative abundance of Lactobacillus species gradually increased from 0 to $9 \mathrm{~h}$, reaching its peak at $9 \mathrm{~h}$ and then decreasing (Figure 7). The abundance of Enterococcus increased gradually throughout the fermentation process, reaching a peak after $24 \mathrm{~h}$ (Figure 7).

In addition, variation in the quantity of particular LAB species level was also analyzed. Lactobacillus helveticus gradually increased from 0 to $9 \mathrm{~h}$ reaching its peak after $9 \mathrm{~h}$ and then decreased (Figure 8). Although Enterococcus faecalis, Enterococcus durans, and Enterococcus casseliflavus increased gradually throughout the 
fermentation process, achieving a maximum after $24 \mathrm{~h}$ (Figure 8), their overall abundance was still quite low. Enterococcus genera, especially Ent. faecalis (which is an opportunist pathogen), can survive at a wide range of pH levels (from 3.5 to 11), and when exposed to mild acidity becomes acid-habituated (Flahaut et al., 1996). The $\mathrm{pH}$ of the koumiss decreased to 3.8 after $9 \mathrm{~h}$ of fermentation (data not shown), allowing Enterococcus species to survive, although in relatively low numbers. Enterococci also are isolated as essential microflora from natural whey cultures used as starter in the manufacture of Mozzarella cheese produced by traditional technology from water buffalo milk (Villani and Coppola, 1994). In dairy products, enterococci serve as an important contributor in ripening and flavor enhancement of several types of food such as cheese and sausages (Giraffa, 2003; Cocolin et al., 2007). However, it is known that some species of enterococci are opportunist pathogens; thus, we believe it is important to keep a balance between Lactobacillus and Enterococcus
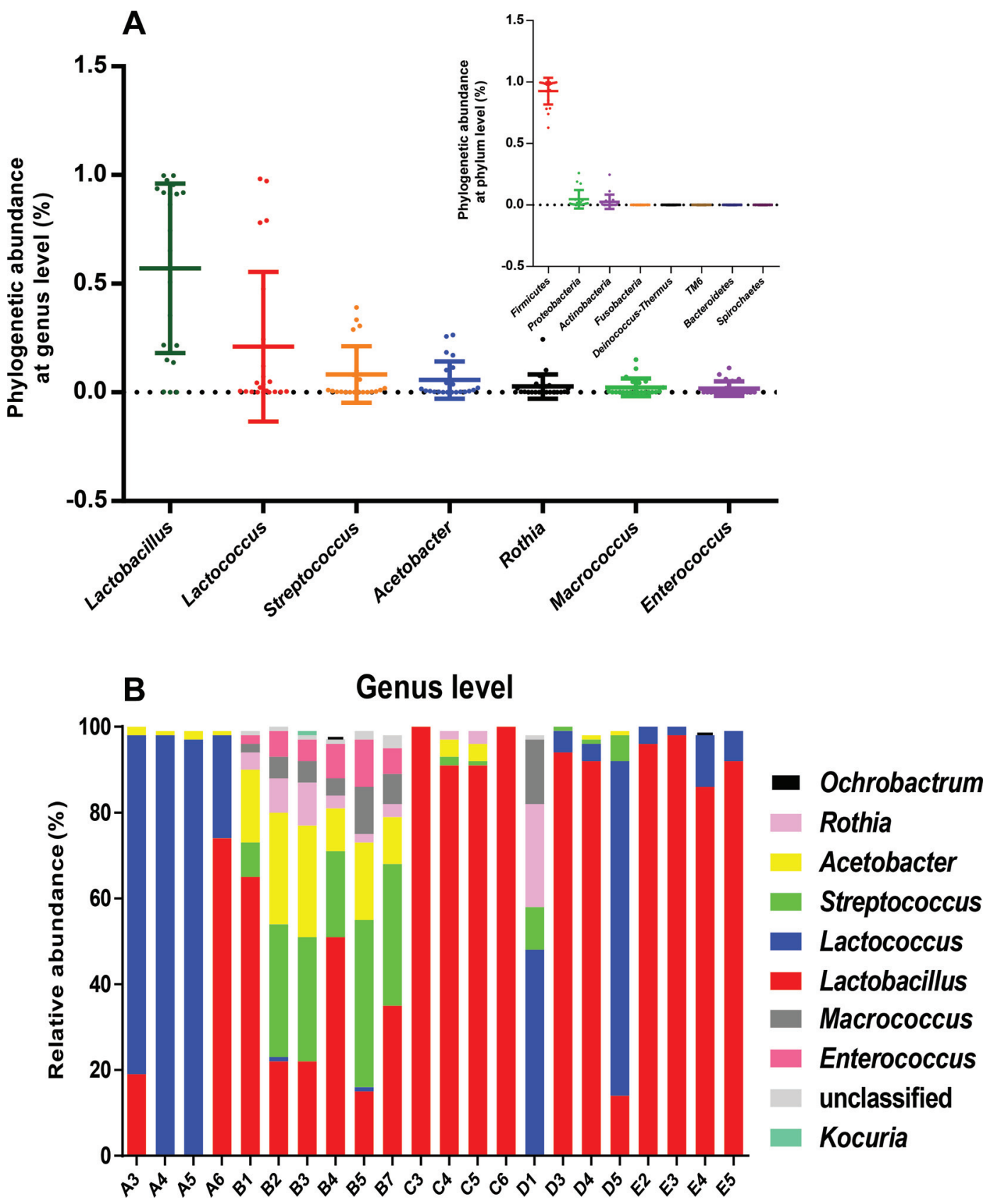

Figure 2. Scatter plot (A) showing the relative abundance and biodiversity at the phylum and genus level; and distribution of different genera (B) in samples from different sites (A to E). Numbers after site designation indicate hours of fermentation. 

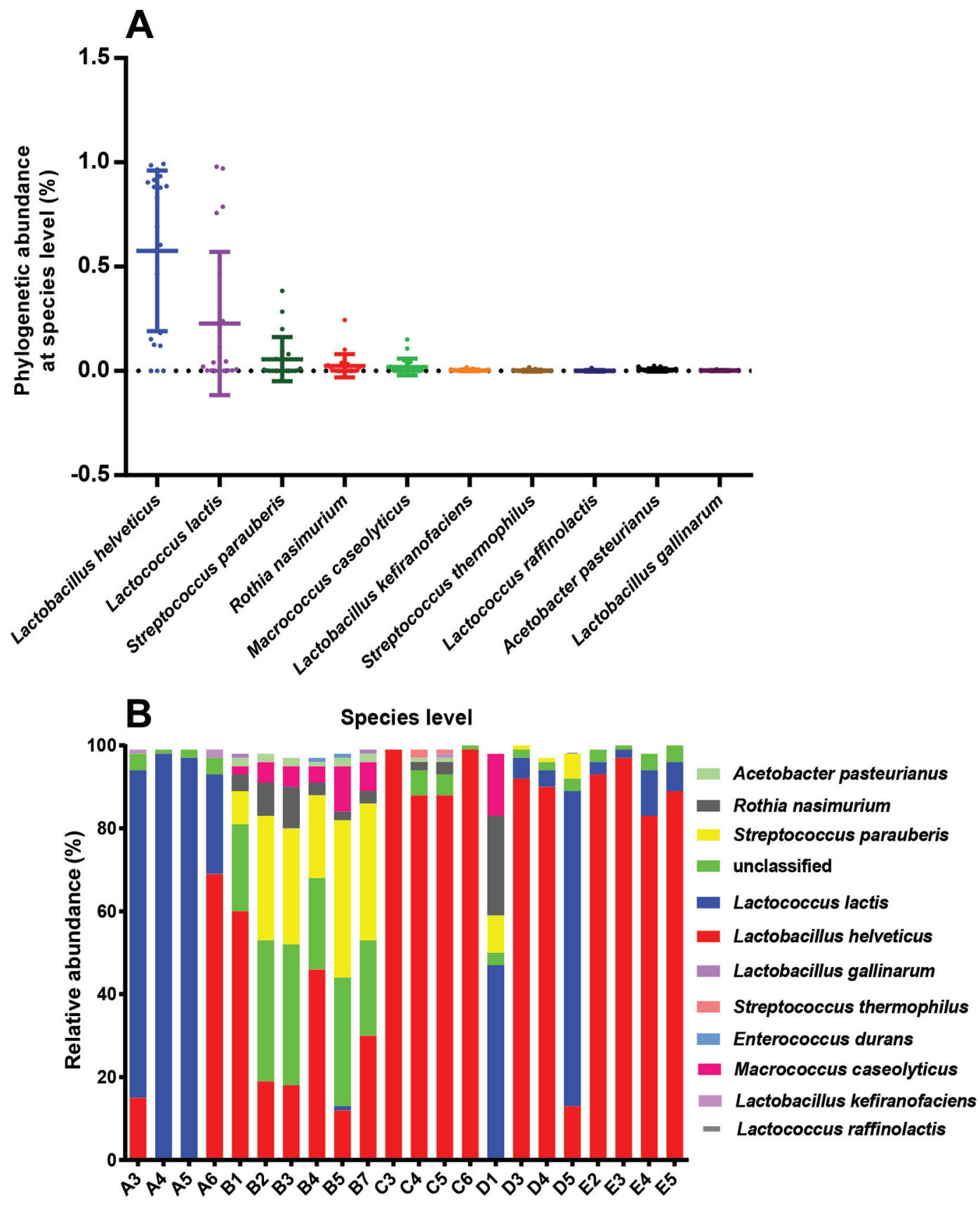

Figure 3. Scatter plot (A) showing the relative abundance and biodiversity at the species level; and distribution of different species (B) in samples from different sites (A to E). Numbers after site designation indicate hours of fermentation.

flora and maintain low levels of Enterococcus species for the safety of naturally fermented koumiss.

In previous studies, many LAB and yeast have been detected in traditional koumiss by culture-dependent and culture-independent methods (Table 3). No differences in microbial composition were found among samples collected from different geographic regions, such as the forest steppe, Gobi Desert, and so on, but significant differences in quantitative ratios of each microbe were observed (Watanabe et al., 2008). Watanabe et al. (2008) also reported that Lb. helveticus, Lactobacillus kefiranofaciens, and Kluyveromyces marxianus were the predominant isolates from koumiss in Mongolia. Lactobacillus helveticus was regarded as predominant species in koumiss collected from Xinjiang, and $L b$. casei was the major microflora of LAB in koumiss from Inner Mongolia (Sun et al., 2010). The isolates from koumiss consisted of mainly Lb. plantarum and Lc. lactis ssp. 
cremoris by culture-dependent and SDS-PAGE method (An et al., 2004). From the above literature reports, $\mathrm{LAB}$ and yeast were proven to be the main components in koumiss starter. Moreover, the benefits of koumiss are mainly attributable to the indigenous LAB, which also were reported to play a major fermentative role on the aroma, texture, and acidity of this product (Wu et al., 2009). However, little is known about the dynamics of succession in bacterial population of koumiss during its fermentation. Here, we first report our use of the SMRT sequencing method to investigate the dynamic variation of bacterial composition in traditional koumiss during its fermentation. It is interesting that Lactobacillus helveticus gradually increased in abundance

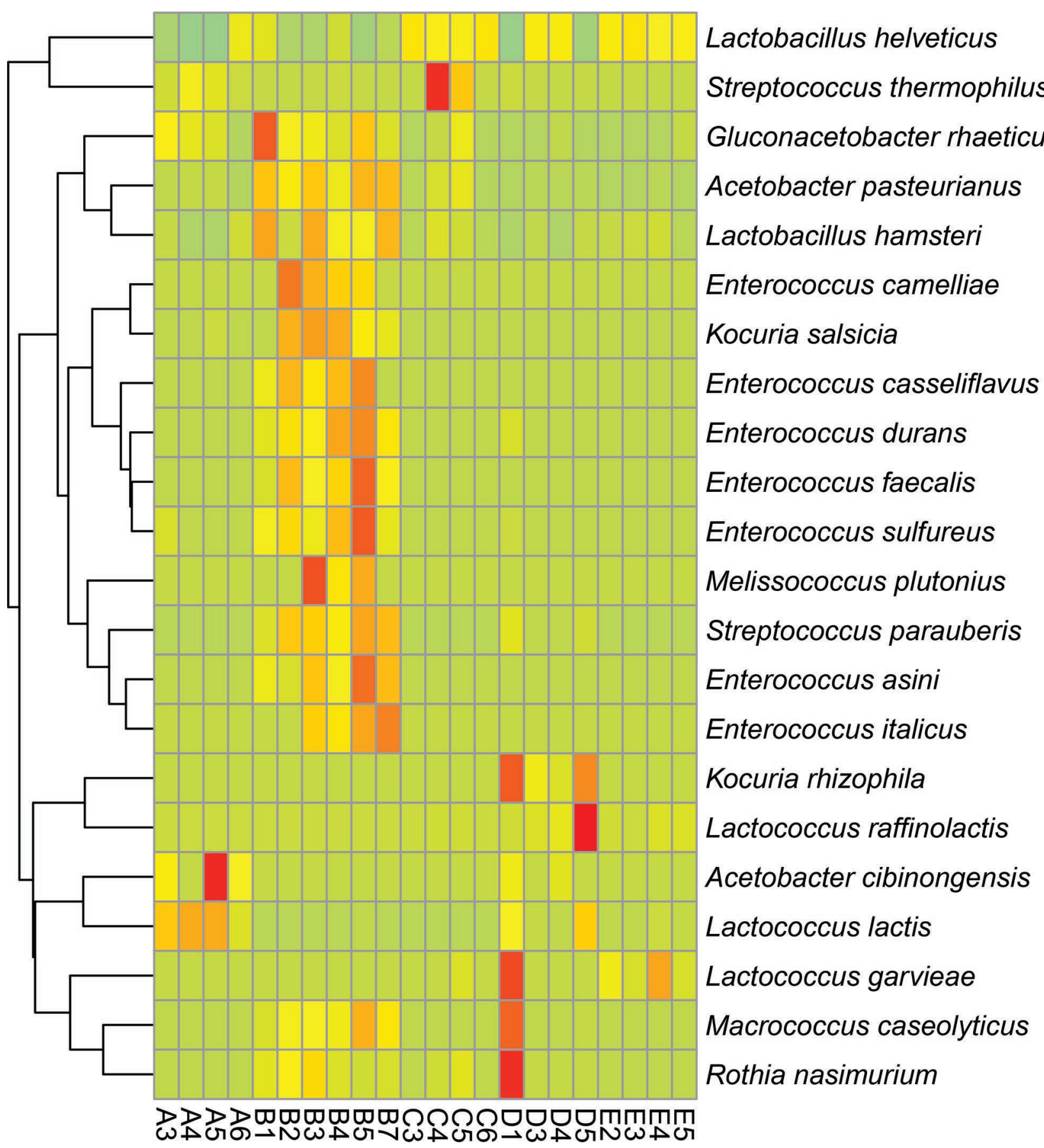

Figure 4. Heat map of bacterial species composition in koumiss from 5 different sample sites. 

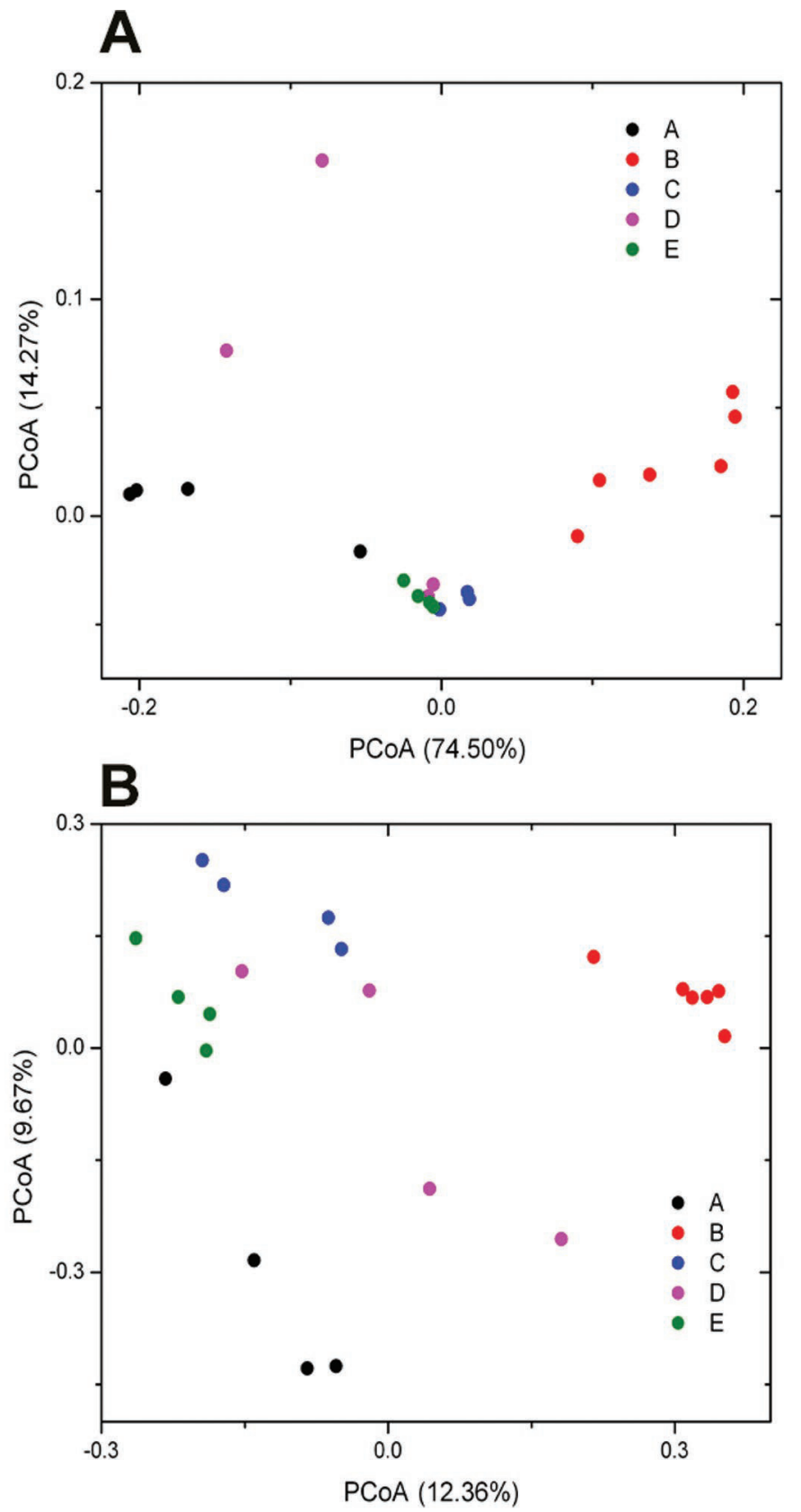

Figure 5. Unifrac principal coordinates analysis of the bacterial communities from 5 different sample sites based on the operational taxonomic units (similarity $\geq 99 \%$ ) using weighted (A) and unweighted analysis (B). PCoA = principal coordinate analysis.

from 0 to $9 \mathrm{~h}$, reaching its peak at $9 \mathrm{~h}$ and then decreasing. Enterococcus gradually increased throughout the fermentation process.

\section{CONCLUSIONS}

To develop new products for industrial application and protect the microbial diversity in artisanal kou- miss, detailed investigation and description of indigenous bacteria present during manufacture and ripening is regarded as a crucial step. In the present study, SMRT sequencing of tagged 16S rRNA gene amplicons was used to obtain a more complete overview on the bacterial community structure during fermentation of traditional koumiss from 5 different sites. One hundred forty-eight species of bacteria were identified from 82 genera and 8 phyla. The predominant species were $L c$. lactis, Streptococcus parauberis, and Lactobacillus helveticus. The results suggested that differences in the species composition of the bacterial community were associated with geographic location. Lactobacillus helveticus gradually increased in abundance from 0 to 9 $\mathrm{h}$, reaching its peak at $9 \mathrm{~h}$ and then decreasing. Enterococcus faecalis, Ent. durans, and Ent. casseliflavus also increased gradually throughout the fermentation process, reaching a maximum after $24 \mathrm{~h}$. It is suggested that Enterococcus maintains a low level in aged koumiss and during its fermentation period compared with Lactobacillus.

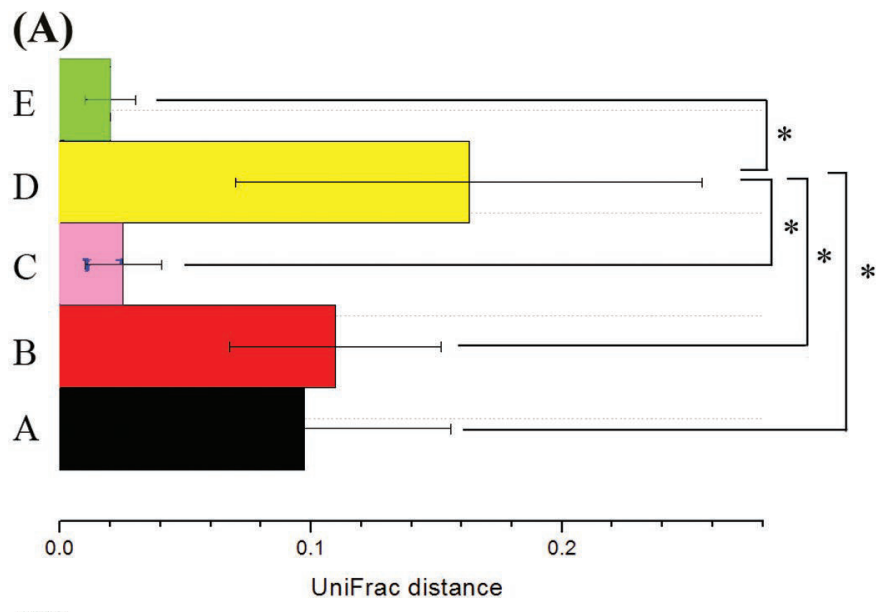

(B)

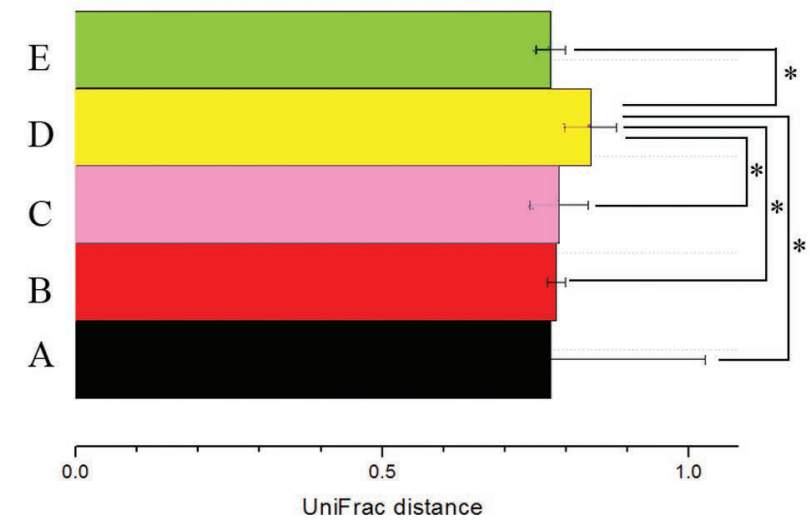

Figure 6. Analysis of bacterial community based on weighted (A) and unweighted (B) Unifrac analysis. ${ }^{*} P<0.05$. 


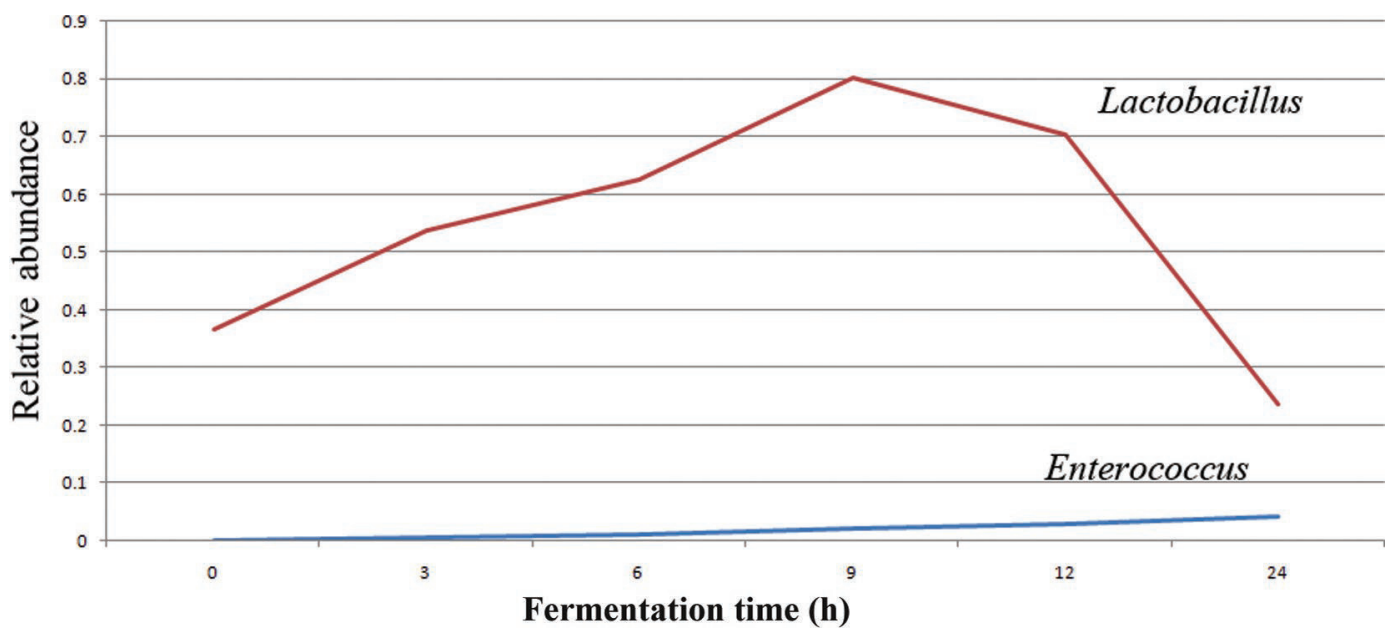

Figure 7. Change in relative abundance of Lactobacillus and Enterococcus species during koumiss fermentation. Color version available online.

\section{ACKNOWLEDGMENTS}

This research was supported by the National Natural Science Foundation of China (Grant No. 31301518), International S\&T Cooperation Program of China
(ISTCP, Grant No. 2014DFR31150), Inner Mongolia Natural Science Foundation (Grant No. 2016MS0316), and Major Program in Inner Mongolia (Grant No. 20140125).
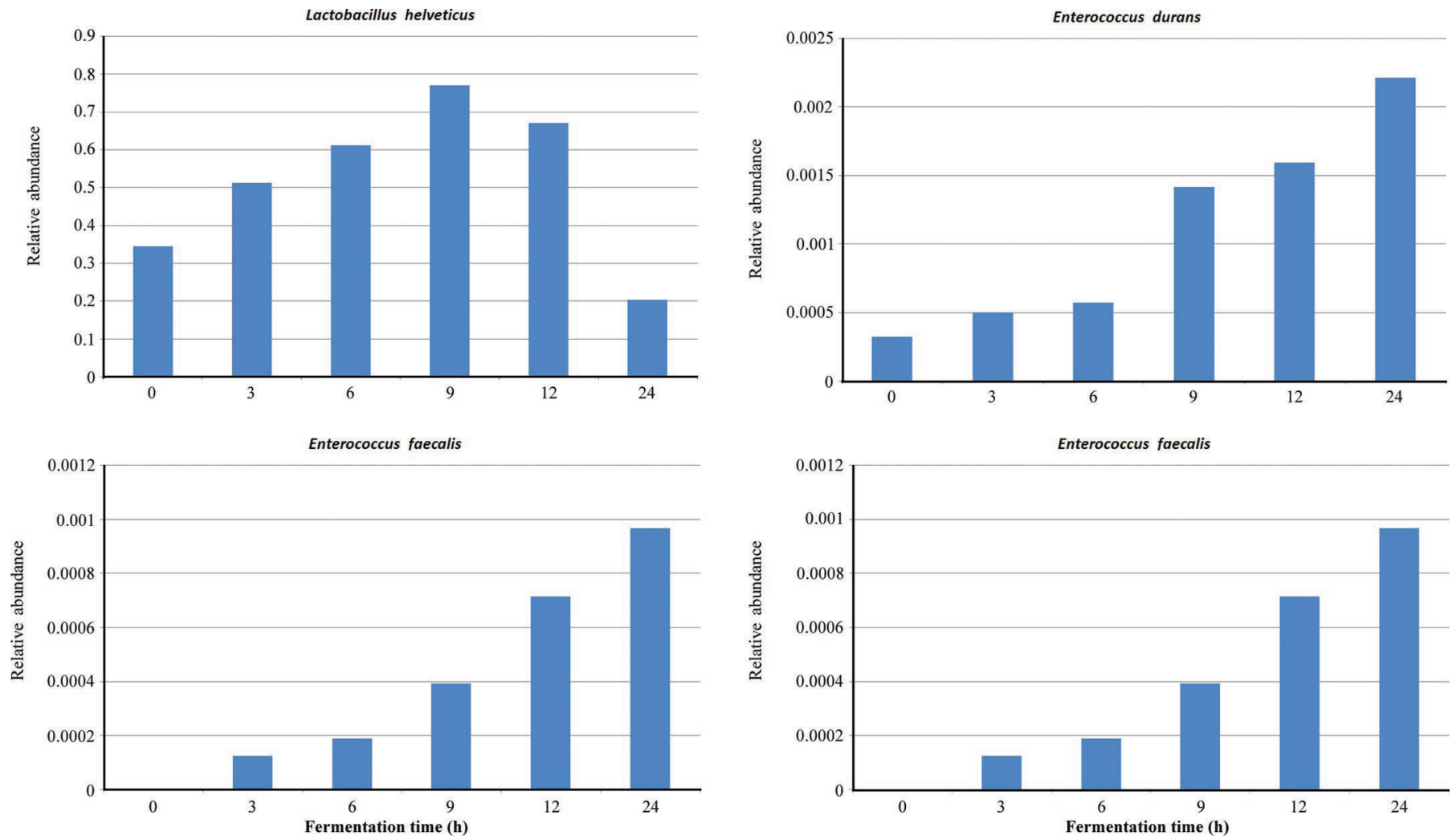

Figure 8. Change in abundance of different species of lactic acid bacteria during koumiss fermentation. Color version available online. 
Table 3. Comparison of composition of lactic acid bacteria and yeast in koumiss from different locations

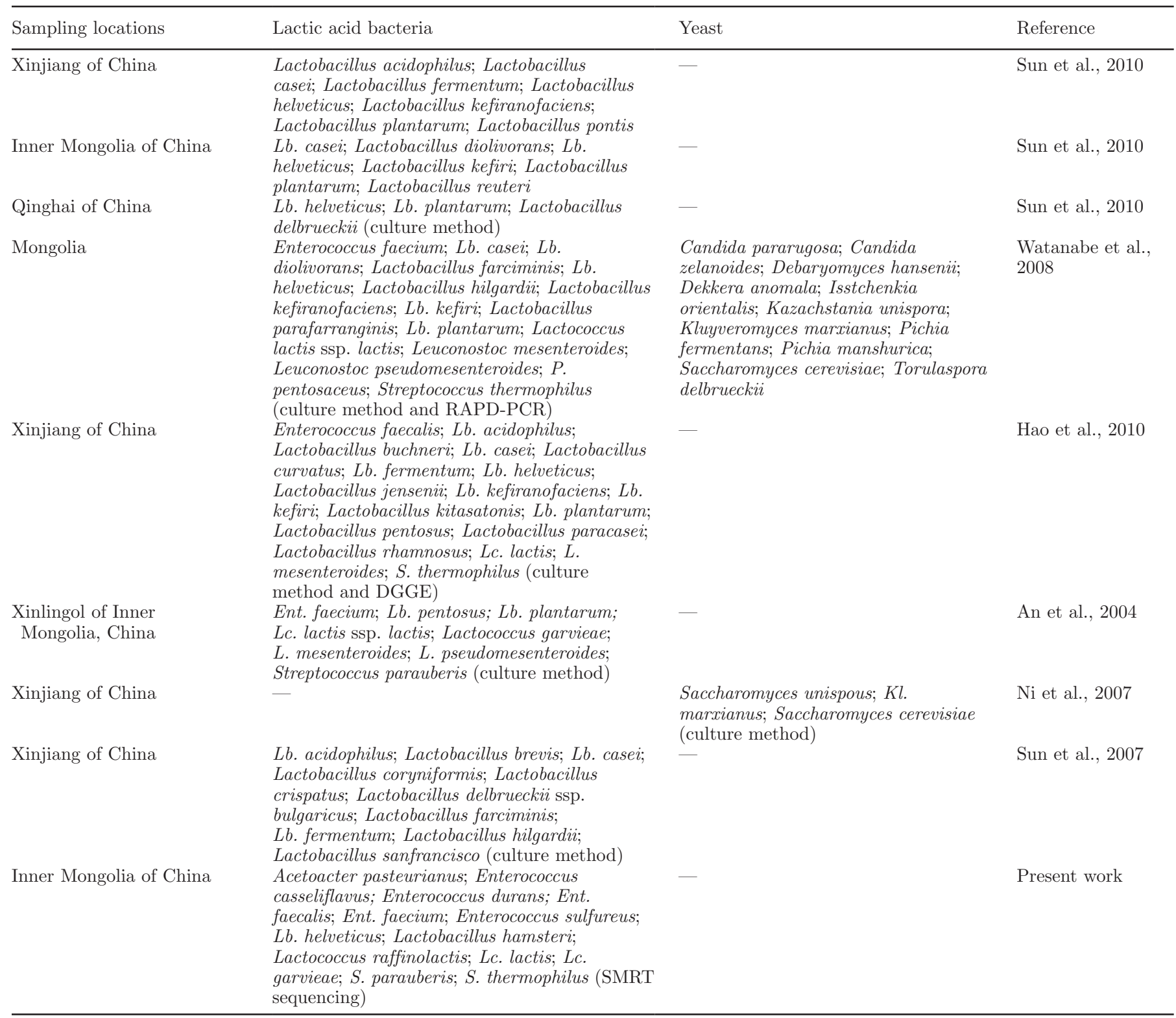

$\overline{{ }^{1} \mathrm{RAPD}}=$ random amplified polymorphic DNA; DGGE = denaturing gradient gel electrophoresis; SMRT = single molecule real-time sequencing technology.

\section{REFERENCES}

ADMI. 1971. Standards for grades of dry milk including methods of analysis. Bulletin 1971:916.

Amir, A., A. Zeisel, O. Zuk, M. Elgart, S. Stern, O. Shamir, P. J. Turnbaugh, Y. Soen, and N. Shental. 2013. High-resolution microbial community reconstruction by integrating short reads from multiple 16S rRNA regions. Nucleic Acids Res. 41:e205.

An, Y., Y. Adachi, and Y. Ogawa. 2004. Classification of lactic acid bacteria isolated from chigee and mare milk collected in Inner Mongolia. Anim. Sci. J. 75:245-252.

Bashir, A., A. A. Klammer, W. P. Robins, C. S. Chin, D. Webster, E. Paxinos, D. Hsu, M. Ashby, S. Wang, P. Peluso, R. Sebra, J. Sorenson, J. Bullard, J. Yen, M. Valdovino, E. Mollova, K. Luong, S. Lin, B. LaMay, A. Joshi, L. Rowe, M. Frace, C. L. Tarr, M. Turnsek, B. M. Davis, A. Kasarskis, J. J. Mekalanos, M. K. Wal- dor, and E. E. Schadt. 2012. A hybrid approach for the automated finishing of bacterial genomes. Nat. Biotechnol. 30:701-707.

Bounaadja, L., D. Albert, B. Chénais, S. Hénault, M. S. Zygmunt, S. Poliak, and B. Garin-Bastuji. 2009. Real-time PCR for identification of Brucella spp.: A comparative study of IS711, bcsp31 and per target genes. Vet. Microbiol. 137:156-164.

Chaves-López, C., R. Tofalo, A. Serio, A. Paparella, G. Sacchetti, and G. Suzzi. 2012. Yeasts from Colombian Kumis as source of peptides with angiotensin I converting enzyme (ACE) inhibitory activity in milk. Int. J. Food Microbiol. 159:39-46.

Chen, Y., Z. Wang, X. Chen, Y. Liu, H. Zhang, and T. Sun. 2010. Identification of angiotensin I-converting enzyme inhibitory peptides from koumiss, a traditional fermented mare's milk. J. Dairy Sci. 93:884-892.

Cocolin, L., R. Foschino, G. M. Comi, and G. Fortina. 2007. Description of the bacteriocins produced by two strains of Enterococcus 
faecium isolated from Italian goat milk. Food Microbiol. 24:752758.

Desai, C., H. Pathak, and D. Madamwar. 2010. Advances in molecular and "-omics" technologies to gauge microbial communities and bioremediation at xenobiotic/anthropogen contaminated sites. Bioresour. Technol. 101:1558-1569.

Devereux, R., and S. S. Wilkinson. 2004. Amplification of ribosomal RNA sequences. Molecular Microbial Ecology Manual, 2nd ed. 3:509-522.

Faveri, M., L. C. Figueiredo, J. A. Shibli, P. J. Pérez-Chaparro, and M. Feres. 2015. Microbiological diversity of peri-implantitis biofilms. Adv. Exp. Med. Biol. 830:85-96.

Flahaut, S., A. Hartke, J. C. Giard, A. Benachour, P. Boutibonnes, and Y. Auffray. 1996. Relationship between stress response toward bile salts, acid and heat treatment in Enterococcus faecalis. FEMS Microbiol. Lett. 138:49-54.

Fuka, M. M., S. Wallisch, M. Enge, G. Welz, J. Havranek, and M. Schloter. 2013. Dynamics of bacterial communities during the ripening process of different Croatian cheese types derived from raw ewe's milk cheeses. PLoS ONE 8:e80734.

Giraffa, G. 2003. Functionality of Enterococci in dairy products. Int. J. Food Microbiol. 88:215-222.

Hao, Y., L. Zhao, H. Zhang, Z. Zhai, Y. Huang, X. Liu, and L. Zhang. 2010. Identification of the bacterial biodiversity in koumiss by denaturing gradient gel electrophoresis and species-specific polymerase chain reaction. J. Dairy Sci. 93:1926-1933.

Humblot, C., and J. P. Guyot. 2009. Pyrosequencing of tagged 16S rRNA gene amplicons for rapid deciphering of the microbiomes of fermented foods such as pearl millet slurries. Appl. Environ. Microbiol. 75:4354-4361.

Ishii, S., and K. Samejima. 2001. Feeding rats with koumiss suppresses the serum cholesterol and triglyceride levels. Milk Science 50:113116.

Justé, A., B. P. H. J. Thommad, and B. Lievens. 2008. Recent advances in molecular techniques to study microbial communities in foodassociated matrices and processes. Food Microbiol. 25:745-761.

Mosher, J. J., E. L. Bernberg, O. Shevchenko, J. Kan, and L. A. Kaplan. 2013. Efficacy of a 3rd generation high-throughput sequencing platform for analyses of $16 \mathrm{~S}$ rRNA genes from environmental samples. J. Microbiol. Methods 95:175-181.

Ni, H. J., Q. H. Bao, T. S. Sun, X. Chen, and H. P. Zhang. 2007. Identification and biodiversity of yeasts isolated from Koumiss in Xinjiang of China. Acta Microbiol. Sin. 47:578-582. (Chinese with English abstract).

Oberman, H., and Z. Libudzisz. 1985. Fermented milks. Pages 308-345 in Microbiology of Fermented Foods, 2nd ed. B. J. B. Wood, ed. Elsevier Applied Science Publishers, London, UK.

Park, W., H. Zhang, B. Zhang, and L. Zhang. 2006. Mare milk. Pages 275-296 in Handbook of Milk of Non-Bovine Mammals, 1st ed. Y. W. Park and G. F. W. Haenlein, ed. Blackwell Publishing, UK.

Quigley, L., O. O'Sullivan, T. P. Beresford, R. P. Ross, G. F. Fitzgerald, and P. D. Cotter. 2012. High-throughput sequencing for detection of subpopulations of bacteria not previously associated with artisanal cheeses. Appl. Environ. Microbiol. 78:5717-5723.
Roberts, R. J., M. O. Carneiro, and M. C. Schatz. 2013. The advantages of SMRT sequencing. Genome Biol. 14:405.

Schornsteiner, E., E. Mann, O. Bereuter, M. Wagner, and S. SchmitzEsser. 2014. Cultivation-independent analysis of microbial communities on Austrian raw milk hard cheese rinds. Int. J. Food Microbiol. 180:88-97.

Sun, T. S., J. G. Wang, L. B. Zhang, B. G. Menghe, and H. P. Zhang. 2007. The biodiversity of lactic acid bacteria isolated from koumiss-A traditional fermented mare milk products of China. Microbiology China 34:451-455. (in Chinese with English abstract).

Sun, Z., W. Liu, Q. Bao, J. Zhang, Q. Hou, L. Kwok, T. Sun, and H. Zhang. 2014. Investigation of bacterial and fungal diversity in tarag using high-throughput sequencing. J. Dairy Sci. 97:60856096 .

Sun, Z., W. Liu, J. Zhang, J. Yu, W. Zhang, C. Cai, B. Menghe, T. Sun, and H. Zhang. 2010. Identification and characterization of the dominant lactobacilli isolated from koumiss in China. J. Gen. Appl. Microbiol. 56:257-265.

Thompson, J., and M. D. Leamnington. 1879. The value of koumiss in wasting diseases. BMJ 1:270.

Uchida, K., M. Hirata, H. Motoshima, T. Urashima, and K. Arai. 2007. Microbiota of 'airag,' 'tarag' and other kinds of fermented dairy products from nomads in Mongolia. Anim. Sci. J. 78:650-658.

Villani, F., and S. Coppola. 1994. Selection of enterococcal strains for water-buffalo Mozzarella cheese manufacture. Ann. Microbiol. Enzimol. 44:97-105.

Wareth, G., F. Melzer, M. C. Elschner, H. Neubauer, and U. Roesler. 2014. Detection of Brucella melitensis in bovine milk and milk products from apparently healthy animals in Egypt by real-time PCR. J. Infect. Dev. Ctries. 8:1339-1343.

Watanabe, K., J. Fujimoto, M. Sasamoto, J. Dugersuren, T. Tumursuh, and S. Demberel. 2008. Diversity of lactic acid bacteria and yeasts in Airag and Tarag, traditional fermented milk products of Mongolia. World J. Microbiol. Biotechnol. 24:1313-1325.

Wszolek, M., B. Kupiec-Teahan, H. S. Guldager, and A. Y. Tamime. 2006. Production of kefir, koumiss and other related products. Pages $174-175$ in Fermented Milks, 1st ed. A. Y. Tamime, ed. Blackwell Publishing, UK.

Wu, R., L. Wang, J. Wang, H. Li, B. Menghe, J. Wu, M. Guo, and H. Zhang. 2009. Isolation and preliminary probiotic selection of lactobacilli from koumiss in Inner Mongolia. J. Basic Microbiol. 49:318-326.

Zhang, W., Z. Sun, B. Menghe, and H. Zhang. 2015. Single molecule, real-time sequencing technology revealed species-and strain-specific methylation patterns of 2 Lactobacillus strains. J. Dairy Sci. 98:3020-3024.

Zhang. W. Y., and H. P. Zhang. 2012. Fermentation and koumiss. Pages 165-171 in Handbook of Animal Based Fermented Food and Beverage Technology, 2nd ed. Y. H. Hui and E. Özgül Evranuz, ed. CRC Press.

Zuo, F. L., X. J. Feng, L. L. Chen, and S. W. Chen. 2014. Identification and partial characterization of lactic acid bacteria isolated from traditional dairy products produced by herders in the western Tianshan Mountains of China. Lett. Appl. Microbiol. 59:549-556. 